\title{
X-ray sources and their optical counterparts in the globular cluster M 22
}

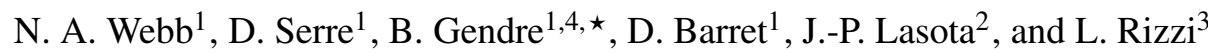 \\ ${ }^{1}$ Centre d'Étude Spatiale des Rayonnements, 9 avenue du Colonel Roche, 31028 Toulouse Cedex 04, France \\ e-mail: Natalie.Webb@cesr.fr \\ 2 Institut d'Astrophysique de Paris, 98bis boulevard Arago, 75014 Paris, France \\ 3 INAF, Osservatorio Astronomico di Padova, Vicolo dell'Osservatorio 5, 35122 Padua, Italy \\ ${ }^{4}$ Istituto di Astrofisica Spaziale e Fisica Cosmica, CNR, via Fosso del Cavaliere, Roma, Italy
}

Received 5 March 2004 / Accepted 26 May 2004

\begin{abstract}
Using XMM-Newton EPIC imaging data, we have detected 50 low-luminosity X-ray sources in the field of view of M 22, where $5 \pm 3$ of these sources are likely to be related to the cluster. Using differential optical photometry, we have identified probable counterparts to those sources belonging to the cluster. Using X-ray spectroscopic and timing studies, supported by the optical colours, we propose that the most central X-ray sources in the cluster are cataclysmic variables, millisecond pulsars, active binaries and a blue straggler. We also identify a cluster of galaxies behind this globular cluster.
\end{abstract}

Key words. Galaxy: globular clusters: individual: M 22 - X-rays: general - stars: binaries: general - galaxies: clusters: general

\section{Introduction}

It is expected that globular clusters (GCs) should contain many binary systems, due to interactions occurring within the clusters (e.g. Di Stefano \& Rappaport 1994; Rasio et al. 2000; Portegies-Zwart et al. 1997), and that these systems could play a critical role in the dynamical evolution of GCs, serving as an internal energy source which counters the tendency of cluster cores to collapse (see Hut et al. 1992, for a review). However, these binaries are difficult to locate, because of crowding in optical observations. The binaries, which are also visible at high energies, can be located using X-ray observations, where the crowding is less severe. Indeed the small population of bright $\mathrm{X}$-ray sources in globular clusters $\left(L_{\mathrm{x}}>10^{36} \mathrm{erg} \mathrm{s}^{-1}\right)$, known to be X-ray binaries (Hertz \& Grindlay 1983), were detected primarily through their X-ray bursts. However, there is also a population of low-luminosity $\left(L_{\mathrm{x}} \lesssim 10^{34.5} \mathrm{erg} \mathrm{s}^{-1}\right.$, Hertz \& Grindlay 1983; Verbunt 2001) X-ray sources. Thanks to the new generation of X-ray observatories, e.g. XMM-Newton and Chandra, the number of sources belonging to this population has rapidly increased. The nature of most these faint X-ray sources can only be determined using follow-up optical observations. A variety of objects have been identified, including many binary systems, such as: X-ray binaries (e.g. Gendre et al. 2003b; Rutledge et al. 2002); cataclysmic variables (e.g. Carson et al. 2000; Gendre et al. 2003a); millisecond pulsars (e.g. Grindlay et al. 2001; Camilo et al. 2000); active binaries (e.g. Kaluzny et al. 1996; Carson et al. 2000); as well as

\footnotetext{
^ Present address
}

some fore- and background objects, e.g. stars (e.g. Gendre et al. 2003a) or clusters of galaxies (e.g. Hertz \& Grindlay 1983).

As a result of these recent identifications, we are now also able to start to constrain the evolutionary paths of these binaries in GCs (e.g. the formation of neutron star low mass X-ray binaries Gendre et al. 2003b; Pooley et al. 2003) and thus not only begin to comprehend the evolution of these systems, but also understand the role played by binaries in countering the core-collapse of GCs. We have therefore obtained X-ray data of the central 30' of the globular cluster M 22 (NGC 6656) with $X M M-N e w t o n$ to detect the low-luminosity $\mathrm{X}$-ray sources in this globular cluster. This GC is one of the closest $(2.6 \pm 0.3 \mathrm{kpc}$ Peterson \& Cudworth 1994) and thus it has previously been chosen as a target by the X-ray satellites Rosat and Einstein (see e.g. Verbunt 2001, and references therein). Eight X-ray sources $\left(L_{\mathrm{x}} \lesssim 10^{34.5} \mathrm{erg} \mathrm{s}^{-1}\right.$, Hertz \& Grindlay 1983), were detected in the direction of the cluster (Johnston \& Verbunt 1994), using the Rosat PSPC. The source detected within the core radius (X4/B) was associated with the cluster. This source has been noted to be somewhat variable, varying by a factor 3 in flux between Rosat observations. The eight sources detected by Rosat include two of the four X-ray sources detected by Einstein (Hertz \& Grindlay 1983). Using the XMM-Newton MOS data only, we detected a total of 34 sources (Webb et al. 2002), including three sources within the core radius, where at least two of these are likely to be associated with the cluster. Here we present both the MOS and the PN data.

There have been several optical observing campaigns carried out on this cluster, however there has been very 
little photometric data taken at the blue end of the spectrum. Pietrukowicz \& Kaluzny (2003), Kaluzny \& Thompson (2001), Clement et al. (2001) have identified a variety of variable stars in M 22, including SX Phe stars, RR Lyr stars and candidate eclipsing binaries. Monaco et al. (2004) have recently carried out wide field photometry in the $B$-, $V$ - and $I$-bands of M 22 , which they have presented in conjunction with some $\mathrm{H}_{\alpha}$ data and $J$-, $H$ - and $K$-band data from the 2 MASS survey to characterise the evolved stellar sequences. They also identify on their colour-magnitude diagrams, previously identified variable stars in this globular cluster. Anderson et al. (2003) have recently identified a new variable star in the centre of M 22 . From its variability, its $\mathrm{H}_{\alpha}$ emission and its coincidence with the Einstein/Rosat source X4/B, they conclude that this source is one of a very small number of confirmed or probable dwarf nova eruptions seen in globular clusters and the first to be found in such a low-concentration cluster.

Using the good astrometry of the X-ray sources, we use differential optical photometry ( $U, B$ and $V$ filters) of the same field of view as the X-ray data, to identify blue (hot) sources that fall within the error circle of the the X-ray sources. Optical sources that are bluer than the main sequence stars of the cluster are likely to be interacting binaries but also cooling white dwarfs, millisecond pulsars or blue stragglers and thus the optical counterparts of the X-ray sources. Also, taking advantage of the spectral and timing information of the brightest X-ray sources we determine the nature of a variety of low-luminosity $\mathrm{X}$-ray sources in the field of M 22.

\section{Observations and data reduction}

\subsection{X-ray data}

We obtained 37 kiloseconds (ks) of EPIC MOS and $34 \mathrm{ks}$ of EPIC PN data of the globular cluster M 22 (NGC 6656), with XMM-Newton. However, 14 ks were affected by high background activity (soft proton flare). Observations were made on September 19-20, 2000, during the "Routine Observing Phase", in the full frame mode (Turner et al. 2001), with the medium filter. Some of these data (MOS) have been presented previously in Webb et al. (2002), but due to a problem with the "Attitude History File", the PN data could not be processed. This problem has now been rectified and we present both the MOS and the PN data, reduced with version 5.4.1 of the XMM-Newton SAS (Science Analysis Software).

The MOS data were reduced using "emchain" with "embadpixfind" to detect the bad pixels. The event lists were filtered, so that $0-12$ of the predefined patterns (single, double, triple, and quadruple pixel events) were retained and the high background periods were identified by defining a count rate threshold above the low background rate and the periods of higher background counts were then flagged in the event list. We also filtered in energy. We used the energy range $0.2-10.0 \mathrm{keV}$, as recommended in the document "EPIC Status of Calibration and Data Analysis" (Kirsch et al. 2002).

The PN data were reduced using the "epchain" of the SAS. Again the event lists were filtered, so that $0-4$ of the predefined patterns (single and double events) were retained, as these have the best energy calibration. We also filtered in energy, where we used the energy range $0.5-10.0 \mathrm{keV}$.

The source detection was done in the same way as Gendre et al. (2003a,b), but briefly we used the SAS wavelet detection algorithm on the $0.5-5.0 \mathrm{keV}$ image, as the signal-tonoise ratio was best in this range. We kept only those sources detected with two or more cameras, with the exception of sources found with the PN camera, which is more sensitive than the two MOS cameras. To improve the astrometry, we used the 3 sources that were detected both in our data and by the Rosat HRI (sources 36, 30 and 60, Table 1). An IDL routine was used to derive the adjustment required for the MOS data to align with the HRI data. We found that we required both a small transversal shift and rotation, in the same manner as Hasinger et al. (2001). This leads to a residual error of $~ 5.6^{\prime \prime}$, where the largest error is due to the position error of the sources detected by the HRI. We detected 50 sources with a maximum likelihood greater than $4.5 \sigma$, which are given in Table 1 , along with their position and count rate. We also give the identification number for the sources detected with only the MOS cameras in Webb et al. (2002) and the sources detected by Einstein and Rosat. The positional error is the $90 \%$ confidence mean statistical error on the position of the XMM-Newton sources convolved with the mean statistical error on the position of the Rosat sources (Verbunt 2001). The majority of these sources can be seen in the contour plot of the central regions of M 22, see Fig. 1.

\subsection{Optical data}

Schott glass $U-, B$ - and $V$-filter photometry was taken with the Wide Field Imager (WFI) mosaic at the $3.9 \mathrm{~m}$ Anglo Australian Telescope (AAT) on September 15-16, 2001. The camera consists of eight $2000 \times 1000$ pixel CCDs, in a square array of $2 \times 4$ detectors, which are mounted at the tripletcorrected $\mathrm{f} / 3.3$ prime focus of the AAT on the prime focus unit, which results in a field of view (FOV) of $33^{\prime} \times 33^{\prime}$, similar to the XMM-Newton FOV. Sky conditions were photometric on the second night. Standard star fields taken from Landolt (1992) were also observed on 2001 September 13 for calibration purposes.

Each image was bias-subtracted and then flat-fielded with twilight sky flats, using the IRAF software (Tody 1986, 1993). The long $U$-band observations were also dark subtracted, as the dark-current was non-negligible in these longer exposures. The data were astrometrically calibrated using the IRAF package MSCRED (Valdes 1998) and the package Wide Field Padova REDuction (WFPRED) developed at the Padova Astronomical Observatory (Held et al., in preparation) and the standard star fields. A refinement to the astrometry was then made using the UCAC (USNO CCD Astrograph Catalog) (Zacharias et al. 2000) of the M 22 data, so that the positions are good to less than $0.1^{\prime \prime}$. The data for each filter were then stacked to create a deep image and the photometry was carried out using DAOPHOT/ALLSTAR (Stetson 1987). The instrumental magnitudes were corrected using the standard stars. We find 123220 stars detected in the $U$-band and at least one of 
Table 1. X-ray sources in the direction of M22, as determined from the EPIC observations, $0.5-10.0 \mathrm{keV}$ band. The identification number, any former identifications and position are given, along with the error on this position and the count rate.

\begin{tabular}{|c|c|c|c|c|c|}
\hline \multirow[b]{2}{*}{ ID } & \multirow{2}{*}{$\begin{array}{l}\text { For. } \\
\text { ID }\end{array}$} & RA (2000) & Dec (2000) & \multirow{2}{*}{$\begin{array}{c}\text { Error } \\
\prime \prime\end{array}$} & \multirow{2}{*}{$\begin{array}{c}\text { Count s }{ }^{-1} \\
\times 10^{-3}\end{array}$} \\
\hline & & & & & \\
\hline 5 & 24 & 183608.28 & -235913.12 & 6.04 & $6.09 \pm 1.04$ \\
\hline 7 & 6 & 183556.82 & -234927.69 & 6.63 & $5.30 \pm 0.89$ \\
\hline 8 & 5 & 183634.79 & -234929.62 & 6.24 & $11.65 \pm 1.29$ \\
\hline 9 & & 183539.66 & -235650.22 & 7.99 & $4.73 \pm 0.98$ \\
\hline 11 & & 183529.99 & -235844.97 & 7.02 & $6.40 \pm 1.14$ \\
\hline 12 & 1 & 183623.97 & -240224.67 & 6.16 & $8.30 \pm 1.21$ \\
\hline 14 & & 183640.67 & -234050.49 & 6.22 & $20.66 \pm 2.56$ \\
\hline 15 & $\mathrm{~b}$ & 183614.28 & -234147.36 & 5.78 & $22.94 \pm 2.94$ \\
\hline 16 & 3 & 183615.69 & -234613.76 & 6.44 & $4.59 \pm 0.75$ \\
\hline 17 & $4,1^{R}$ & 183610.69 & -234629.10 & 5.58 & $13.14 \pm 1.33$ \\
\hline 18 & & 183613.58 & -234837.03 & 6.65 & $3.19 \pm 0.64$ \\
\hline 19 & 8 & 183605.91 & -235050.69 & 5.92 & $7.74 \pm 1.02$ \\
\hline 20 & 9 & 183615.80 & -235058.84 & 6.09 & $4.77 \pm 0.61$ \\
\hline 21 & 10 & 183645.70 & -235159.16 & 5.62 & $10.44 \pm 1.04$ \\
\hline $23^{h}$ & 21 & 183628.61 & -235625.92 & 5.89 & $6.25 \pm 0.83$ \\
\hline 24 & & 183542.39 & -235638.67 & 5.94 & $12.66 \pm 1.80$ \\
\hline 25 & 23 & 183700.91 & -235829.39 & 5.79 & $11.90 \pm 1.49$ \\
\hline 27 & $\mathrm{f}, 7^{R}$ & 183600.62 & -240433.16 & 6.00 & $13.95 \pm 2.32$ \\
\hline 28 & & 183616.91 & -234134.96 & 6.22 & $19.07 \pm 2.42$ \\
\hline 29 & 2 & 183628.08 & -234509.49 & 5.42 & $25.53 \pm 1.93$ \\
\hline 30 & $\mathrm{c}, 3^{R}, \mathrm{~A}$ & 183550.91 & -234656.29 & 5.06 & $79.11 \pm 3.71$ \\
\hline 31 & $\mathrm{~d}$ & 183543.55 & -234821.60 & 5.13 & $46.10 \pm 2.71$ \\
\hline $32^{h}$ & 11 & 183618.67 & -235219.06 & 5.51 & $8.08 \pm 0.88$ \\
\hline $33^{c}$ & 13 & 183621.97 & -235337.65 & 5.37 & $12.03 \pm 1.29$ \\
\hline 34 & 14 & 183639.37 & -235347.47 & 5.47 & $9.23 \pm 0.92$ \\
\hline 35 & & 183529.84 & -235434.33 & 8.79 & $2.73 \pm 1.03$ \\
\hline $36^{c}$ & $16,4^{R}, \mathrm{~B}$ & 183624.97 & -235438.04 & 4.98 & $49.14 \pm 1.81$ \\
\hline 37 & 15 & 183638.80 & -235447.68 & 6.26 & $3.51 \pm 0.52$ \\
\hline 38 & 17 & 183559.23 & -235504.22 & 5.31 & $16.19 \pm 1.31$ \\
\hline $39^{c}$ & 18 & 183625.12 & -235516.97 & 5.06 & $27.73 \pm 1.36$ \\
\hline 40 & 20 & 183602.96 & -235526.42 & 5.42 & $17.92 \pm 1.56$ \\
\hline 41 & 22 & 183555.93 & -235722.44 & 6.09 & $10.31 \pm 1.84$ \\
\hline 42 & & 183635.08 & -235916.15 & 7.83 & $0.78 \pm 0.31$ \\
\hline 43 & 25 & 183659.61 & -240029.76 & 5.37 & $21.46 \pm 1.77$ \\
\hline 44 & 19 & 183618.46 & -240056.14 & 5.32 & $16.03 \pm 1.35$ \\
\hline 45 & $\mathrm{~g}, 8^{R}$ & 183627.71 & -24639.72 & 7.11 & $6.33 \pm 1.14$ \\
\hline 49 & & 183601.47 & -235752.70 & 7.07 & $5.26 \pm 1.03$ \\
\hline $55^{h}$ & & 183614.94 & -235538.91 & 7.14 & $1.92 \pm 0.38$ \\
\hline 59 & $\mathrm{a}$ & 183650.40 & -234140.54 & 5.28 & $64.36 \pm 3.80$ \\
\hline 60 & $27,9^{R}$ & 183649.05 & -234813.43 & 6.02 & $8.27 \pm 1.24$ \\
\hline 63 & & 183651.85 & -240014.54 & 7.86 & $1.52 \pm 0.52$ \\
\hline 66 & & 183722.41 & -235443.35 & 9.54 & $6.14 \pm 1.61$ \\
\hline 67 & & 183658.62 & -234455.76 & 8.41 & $6.78 \pm 1.73$ \\
\hline 70 & & 183542.87 & -235720.08 & 8.19 & $5.00 \pm 1.37$ \\
\hline 71 & & 183625.69 & -235911.89 & 10.40 & $2.58 \pm 0.73$ \\
\hline 72 & & 183601.29 & -234618.50 & 10.10 & $3.66 \pm 1.23$ \\
\hline 73 & & 183621.08 & -234922.20 & 7.55 & $2.32 \pm 0.67$ \\
\hline 75 & & 183710.17 & -235226.63 & 8.72 & $3.44 \pm 1.13$ \\
\hline 76 & & 183652.76 & -235407.03 & 7.97 & $2.60 \pm 0.72$ \\
\hline 77 & & 183652.80 & -235858.18 & 8.78 & $2.62 \pm 0.83$ \\
\hline 78 & & 183544.47 & -240011.75 & 8.43 & $2.56 \pm 1.03$ \\
\hline
\end{tabular}

${ }^{c}$ Sources within the core; ${ }^{h}$ sources within the half mass radius. Former ID: A-B, Einstein, Hertz \& Grindlay (1983).

$1-8^{R}$, Rosat PSPC, Johnston \& Verbunt (1994).

$9^{R}$, Rosat HRI, Verbunt (2001).

1-27, a-g, XMM-Newton MOS only, Webb et al. (2002).

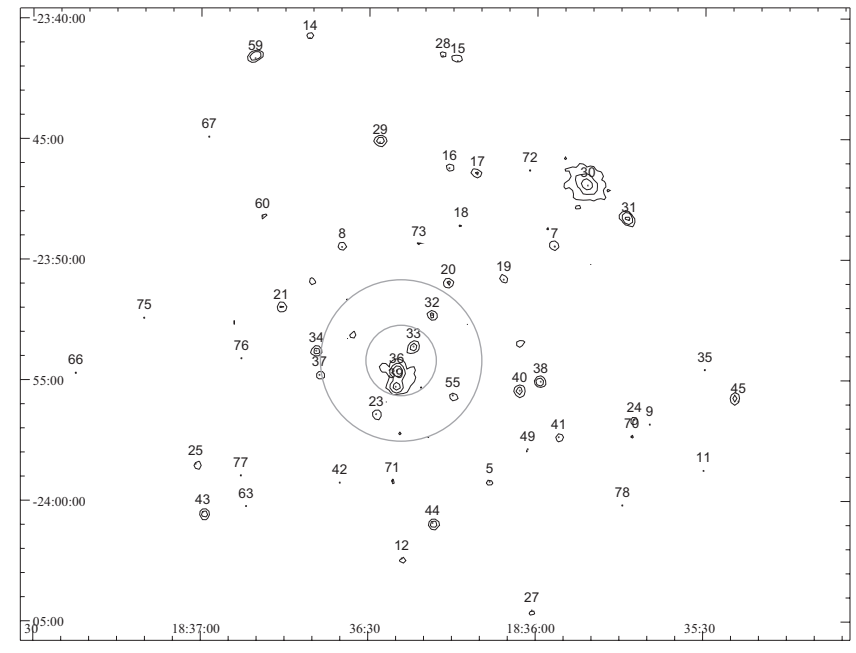

Fig. 1. X-ray contour plot of the central region of M 22. The inner circle shows the core radius and the outer circle shows the half mass radius. The abscissa indicates the right ascension in ${ }^{\mathrm{h}} \mathrm{m} \quad \mathrm{s}$ and the ordinate indicates the declination in ${ }^{\circ}, "$ ". The contours represent 5 , 7 and $14 \sigma$ confidence levels and the source numbers are the same as those found in Table 1 .

the other two bands, where the maximum matching distance was 3 pixels $\left(<1.5^{\prime \prime}\right)$. The magnitudes range from approximately 14.0 to 22.8 in each filter. The photometric errors range from 0.01 to $0.1 \mathrm{mag}$.

\section{The X-ray sources}

We have detected three sources within the core radius $\left(85.11^{\prime \prime}\right.$ Djorgovski 1993), sources 39, 33 and 36 (indicated by a ${ }^{c}$ in Table 1), and a further three sources within the half-mass radius (3.3' Harris 1999) (indicated by a ${ }^{h}$ in Table 1 ). We computed the limit of the detectable flux within the half mass radius, assuming a $0.6 \mathrm{keV}$ blackbody spectral model in the same way as Johnston \& Verbunt (1996). The limiting luminosity is $L_{\mathrm{x}}=4 \times 10^{30} \mathrm{erg} \mathrm{s}^{-1}$ for the PN detector $(0.5-10.0 \mathrm{keV})$ and $L_{\mathrm{x}}=1.2 \times 10^{31} \mathrm{erg} \mathrm{s}^{-1}$ for the MOS detectors at the centre of the field of view.

Many of our detected sources are background sources. We have used the statistical $\log N-\log S$ relationship of extragalactic sources derived from the Lockman Hole (Hasinger et al. 2001) to estimate the background source population. We converted the source count rates to fluxes using a power law model with spectral index of -2 , as Hasinger et al. (2001). We employed the method of Cool et al. (2002) and Gendre et al. (2003a) which takes into account vignetting. We calculated the average limiting flux in four annuli on the PN camera. These annuli were bound by the core radius, the half mass radius, 7.5', and 15'. We expect $1.0 \pm 0.1,3.3 \pm 0.8,13.9 \pm 2$ and $30 \pm 3$ background sources in these annuli respectively where the uncertainty is the $10 \%$ error estimated on the flux, as Hasinger et al. (2001). We find 3, 3, 17, and 27 sources in these annuli respectively. This indicates that $5 \pm 3$ sources belong to the cluster, which are grouped about the centre.

In addition, it is also possible to calculate the probability that the sources within the core are not simply spurious 
identifications with the cluster, in the same manner as Verbunt (2001). Using the probability, $p$, that no serendipitous sources are detected in the PN observation at a distance $r<R$ from the cluster centre, located at RA $=18^{\mathrm{h}} 36^{\mathrm{m}} 24.2^{\mathrm{s}}$, Dec $=$ $-23^{\circ} 54^{\prime} 12^{\prime \prime}$ for M 22 (Djorgovski \& Meylan 1993), where $p=1-\left(R / r_{\mathrm{d}}\right)^{2}$ and $r_{d}$ is the radius of the field of view. The probability of not finding any sources in a single trial simply by chance, within the core radius, is $98.9 \%$. In 50 trials, for the 50 sources, the probability of finding no sources within the core radius is $59.7 \%$. It is therefore possible that the faintest source within the core radius (source 33) may not be related to the cluster, where from their brightness alone, the other two core sources are more likely to be related.

\subsection{Spectral analysis}

Nine X-ray sources, including all three sources within the core radius, have enough counts that we could extract and fit a spectrum. We extracted the spectra using an extraction radius of $\sim 30^{\prime \prime}$ for the core sources (due to their close proximity) and $\sim 45^{\prime \prime}$ for the rest of the sources, with the exception of the extended source, number 30 , where we used an extraction radius of $1^{\prime}$. We used a similar neighbouring surface, free from $\mathrm{X}$-ray sources to extract a background file. We rebinned the MOS data into $15 \mathrm{eV}$ bins and the PN data into $5 \mathrm{eV}$ bins. We used the SAS tasks "rmfgen" and "arfgen" to generate a "redistribution matrix file" and an "ancillary response file", for each source. We binned up the data to contain at least 20 net counts/bin. We then used Xspec (version 11.1.0) to fit the spectra. We initially tried simple models, such as a power law, a blackbody, a bremsstrahlung and a raymond smith fit, which we found to provide a good fit to the data for the majority of the sources. However, we found that for sources 33 and 36 and the extended sources 29 and 30 , we required a more complicated model to fit the data. The results of the spectral fitting can be found in Table 2, where the best fits are given, along with the goodness of fit $\left(\chi_{v}^{2}\right)$ and the flux in the $0.2-10.0 \mathrm{keV}$ range.

\subsection{Variability analysis}

We carried out a variability test on all the sources by dividing the data into two equal frames of $17 \mathrm{ks}$ and then into four equal frames of $8.5 \mathrm{ks}$ and looking for variability in the source counts from frame to frame. However, within the errors, we found no significant variability of any of the sources, unlike Gendre et al. (2003a), who found several variable sources in $\omega$ Centauri, using this method.

We also carried out a variability analysis on the nine sources with enough counts for such a test. These are the sources given in Table 2. We extracted the lightcurves using regions of the same size as those in the spectral analysis (Sect. 3.1). We used the filtered data between $0.2-10.0 \mathrm{keV}$ that was uninterrupted by gaps due to the flare screening. This is important as gaps in the data can bias variability tests. We used the ftool, lcstats $^{1}$ (Blackburn 1995) to perform a

\footnotetext{
${ }^{1}$ http://heasarc.gsfc.nasa.gov/ftools/
}

Kolmogorov-Smirnov probability of constancy test and the $\chi^{2}$ probability of constancy test. The results of these tests, along with the size and number of data bins are given in Table 2. Only one of the sources is significantly variable, one of the core sources, source 39.

\section{The optical counterparts}

We constructed a $U,(U-V)$ colour-magnitude diagram with the sources from the central 12.5' of M 22 (34614 sources), see Fig. 2. Only the central region was used as it was only in this region that we found potential optical counterparts of the $\mathrm{X}$-ray sources (with the exception of source 35 ). The majority of the optical sources in Fig. 2 are likely to be cluster members, due to their centrally located positions, although some of these sources may be foreground stars. However, as all the data were taken at the same time, we have no proper motion estimates for these sources and thus we can not discern the cluster sources from foreground stars. The average $(U-V)$ value of main-sequence stars in M 22 is described by an arc given by $(U-V)=((U / 10.0)-1.3) \pm 0.1(22<U<18)$. We have therefore defined that a star is bluer than the main-sequence if $(U-V)<(U / 10.0)-1.4$. We have identified blue sources that fall in the error circle of the X-ray sources and they can be seen in the colour-magnitude diagram in Fig. 2, where the diamonds indicate a blue optical source found inside the error circle of an X-ray source in the core, the triangles indicate a blue optical source found inside the error circle of an X-ray source in the half mass radius, the squares indicate a blue optical source found inside the error circle of an X-ray source just outside the half mass-radius and the crosses indicate blue optical sources found inside the error circle of an X-ray source way outside the half mass-radius.

We find that eleven X-ray source error circles contain at least one blue optical source. We find potential optical counterparts for one of the core X-ray sources (39), all three X-ray sources in the half-mass radius $(23,32$ and 55) and three of the four X-ray sources that fall just outside the half massradius (20, 21 and 34). We also find potential optical counterparts for three sources a few arcminutes from the centre of the cluster $(5,44$ and 78) and a possible optical counterpart for source 35 , which lies almost $14^{\prime}$ from the centre of the cluster. We do not find an optical counterpart for the two most central sources within the core, but this may be due to the stellar confusion in this densely populated region. In the cases of sources 37 and 39, we find two possible optical counterparts. The positions as well as $U$ and $(U-V)$ magnitudes can be found in Table 3. Some of the astrometric matches between the XMM-Newton $\mathrm{X}$-ray sources and the blue stars maybe chance coincidences. We have calculated the number of blue stars per unit area as a function of the radial distance from the centre of the cluster, in the same way as Edmonds et al. (2003). We have thus been able to calculate the probability of a chance coincidence. We find probabilities of $4 \%, 0.6 \%$ and $0.01 \%$ in the core, half-mass radius and outside the half mass radius.

As some of our X-ray sources could be active binaries, which have red (cool) optical counterparts, not easily detectable with our optical photometry, we have also 
Table 2. In the left hand side of the table, the best fitting models to spectra from the MOS and PN data. Where no error value is given for the $N_{\mathrm{H}}\left(\times 10^{21} \mathrm{~cm}^{-2}\right)$, the $N_{\mathrm{H}}$ was frozen to that of the cluster $\left(2.2 \times 10^{21} \mathrm{~cm}^{-2}\right.$ Johnston \& Verbunt 1994). The flux given is unabsorbed flux $\left(\times 10^{-14} \mathrm{erg} \mathrm{cm}^{-2} \mathrm{~s}^{-1}\right)$ in the $0.2-10.0 \mathrm{keV}$ range, with errors of the order $\pm 10 \%$. In the right hand side of the table, the results from testing for variability of the sources is given. This includes the number of data bins, the length (in seconds) of each data bin and the probabilities from a Kolmogorov-Smirnov probability of constancy test and the $\chi^{2}$ probability of constancy test. Source numbers correspond to those given in Table 1 and ${ }^{m}$ indicates possible cluster members, see Sect. 5.1.

\begin{tabular}{|c|c|c|c|c|c|c|c|c|c|c|c|c|}
\hline $\mathrm{Src}$ & $\begin{array}{c}N_{\mathrm{H}} \\
\times 10^{21} \mathrm{~cm}^{-2} \\
\end{array}$ & Model & $k T(\mathrm{keV})$ & $\begin{array}{l}\text { Photon } \\
\text { Index }\end{array}$ & Abundance & $\bar{z}$ & $\chi_{v}^{2}$ & d.o.f. & Flux & Bins & $\begin{array}{c}\text { Time } \\
\text { (s) }\end{array}$ & $\begin{array}{l}\mathrm{KS} / \\
\chi^{2}\end{array}$ \\
\hline \multirow[t]{5}{*}{$36^{m}$} & $2.29 \pm 0.70$ & PL & - & $1.70 \pm 0.13$ & - & & 1.27 & 39 & 9.8 & 10 & 2000 & $9 \times 10^{-3} /$ \\
\hline & 2.2 & $\mathrm{BB}$ & $0.65 \pm 0.03$ & - & - & & 3.11 & 40 & & & & $3 \times 10^{-5}$ \\
\hline & $1.45 \pm 0.50$ & Brems. & $9.69 \pm 3.11$ & - & - & & 1.30 & 39 & & & & \\
\hline & $1.50 \pm 0.50$ & RS & $8.96 \pm 3.04$ & - & $0.37 \pm 0.53$ & & 1.36 & 37 & & & & \\
\hline & 2.2 & PL+Gau. ${ }^{*}$ & - & $2.03 \pm 0.21$ & - & & 1.08 & 36 & & & & \\
\hline \multirow[t]{4}{*}{$33^{m}$} & $1.4 \pm 2.7$ & PL & - & $1.98 \pm 0.62$ & - & & 0.59 & 11 & 1.7 & 4 & 5000 & $1 \times 10^{-2} /$ \\
\hline & 2.2 & $\mathrm{BB}$ & $0.48 \pm 0.08$ & - & - & & 0.80 & 12 & & & & 0.85 \\
\hline & $1.0 \pm 2.1$ & Brems. & $3.60 \pm 2.90$ & - & - & & 0.58 & 11 & & & & \\
\hline & 2.2 & $\mathrm{RS}+\mathrm{RS}$ & $0.07+3.76^{@}$ & - & - & - & 0.49 & 10 & & & & \\
\hline \multirow[t]{4}{*}{$39^{m}$} & 2.2 & $\mathrm{PL}$ & - & $1.45 \pm 0.12$ & - & & 1.52 & 25 & 5.6 & 10 & 2000 & $2 \times 10^{-10} /$ \\
\hline & 2.2 & $\mathrm{BB}$ & $0.90 \pm 0.06$ & - & - & & 2.80 & 25 & & & & $3 \times 10^{-4}$ \\
\hline & 2.2 & Brems. & $19.98 \pm 13.35$ & - & - & & 1.57 & 25 & & & & \\
\hline & 2.2 & RS & $19.91 \pm 19.17$ & - & $6.7 \pm 2.2 \times 10^{-3}$ & & 1.63 & 24 & & & & \\
\hline \multirow[t]{2}{*}{$32^{m}$} & 2.2 & PL & - & $6.89 \pm 1.63$ & - & & 2.39 & 4 & 0.6 & 4 & 5000 & $2 \times 10^{-7} /$ \\
\hline & 2.2 & $\mathrm{BB}$ & $0.13 \pm 0.02$ & - & - & & 2.37 & 4 & & & & 0.85 \\
\hline \multirow[t]{4}{*}{43} & $5.3 \pm 4.9$ & PL & - & $6.3 \pm 3.45$ & - & & 0.79 & 9 & 3.1 & 4 & 5000 & $6 \times 10^{-3} /$ \\
\hline & 2.2 & BB & $0.15 \pm 0.02$ & - & - & & 0.86 & 10 & & & & 0.86 \\
\hline & $1.1 \pm 2.2$ & Brems. & $0.48 \pm 0.28$ & - & - & & 0.83 & 9 & & & & \\
\hline & $9.3 \pm 4.0$ & RS & $0.18 \pm 0.06$ & - & $2 \pm 64$ & $0.16 \pm 0.4$ & 1.30 & 7 & & & & \\
\hline 44 & 2.2 & PL & - & $1.56 \pm 0.20$ & - & & 4.16 & 6 & 4.2 & 4 & 5000 & $\begin{array}{c}1 \times 10^{-2} / \\
0.85\end{array}$ \\
\hline \multirow[t]{5}{*}{30} & $3.5 \pm 0.4$ & PL & - & $2.71 \pm 0.13$ & - & & 1.73 & 62 & 85.3 & 10 & 2000 & $2 \times 10^{-2} /$ \\
\hline & 2.2 & $\mathrm{BB}$ & $0.39 \pm 0.01$ & - & - & & 3.50 & 63 & & & & $4 \times 10^{-2}$ \\
\hline & $1.9 \pm 0.3$ & Brems. & $1.98 \pm 0.20$ & - & - & & 1.72 & 62 & & & & \\
\hline & $1.88 \pm 0.27$ & $\mathrm{RS}$ & $2.22 \pm 0.20$ & - & $0.45 \pm 017$ & $0.10 \pm 0.01$ & 1.38 & 60 & & & & \\
\hline & $1.9 \pm 0.30$ & MEKAL & $2.25 \pm 0.21$ & - & $0.44 \pm 0.18$ & $0.10 \pm 0.02$ & 1.37 & 59 & & & & \\
\hline \multirow[t]{4}{*}{31} & $0.80 \pm 0.90$ & PL & - & $2.48 \pm 0.44$ & - & & 0.86 & 21 & 30.0 & 10 & 2000 & $2 \times 10^{-6} /$ \\
\hline & 2.2 & BB & $0.23 \pm 0.02$ & - & - & & 1.81 & 22 & & & & 0.19 \\
\hline & 2.2 & Brems. & $0.75 \pm 0.11$ & - & - & & 1.25 & 22 & & & & \\
\hline & $6.5 \pm 3.0$ & $\mathrm{RS}$ & $0.77 \pm 0.16$ & - & $1.00 \pm 3.6$ & $1.57 \pm 0.05$ & 1.70 & 19 & & & & \\
\hline \multirow[t]{5}{*}{29} & $1.70 \pm 0.70$ & PL & - & $1.68 \pm 0.22$ & - & & 0.74 & 18 & 16.0 & 10 & 2000 & $2 \times 10^{-5} /$ \\
\hline & 2.2 & BB & $0.55 \pm 0.04$ & - & - & & 2.54 & 19 & & & & 0.11 \\
\hline & $1.05 \pm 0.53$ & Brems. & $9.89 \pm 6.20$ & - & - & & 0.72 & 18 & & & & \\
\hline & $1.50 \pm 0.54$ & $\mathrm{RS}$ & $5.40 \pm 1.37$ & - & $1.57 \pm 1.15$ & $0.18 \pm 0.09$ & 0.66 & 16 & & & & \\
\hline & $1.44 \pm 0.53$ & MEKAL & $5.77 \pm 1.31$ & - & $1.73 \pm 1.48$ & $0.16 \pm 0.08$ & 0.70 & 15 & & & & \\
\hline
\end{tabular}

$\mathrm{PL}=$ power law; $\mathrm{BB}=$ blackbody; Brems. = bremsstrahlung; $\mathrm{RS}=$ Raymond Smith; Gau. = Gaussian line.

${ }^{*}$ Line centre $=1.00 \pm 0.05, \sigma=0.09 \pm 0.06 .{ }^{@}$ Errors are \pm 0.02 and \pm 0.04 respectively.

cross-correlated several catalogues that contain known variable stars in M 22 (Pietrukowicz \& Kaluzny 2003; Kaluzny \& Thompson 2001; Clement et al. 2001). However, none of the variable stars in these catalogues fall within, or even close to, the error circles of our X-ray sources. The possible dwarf nova, detected by Anderson et al. (2003) does fall within the error circle of our X-ray source 36, which is Rosat source 4. We discuss the nature of the X-ray source in Sect. 5.1.1.

\section{Discussion}

\subsection{Possible cluster members with fitted spectra}

\subsubsection{Source 36}

This is the most centrally located source in the globular cluster M 22. Its absorption, see Table 2, is consistent with that of the globular cluster. The X-ray spectrum is well fitted by a 


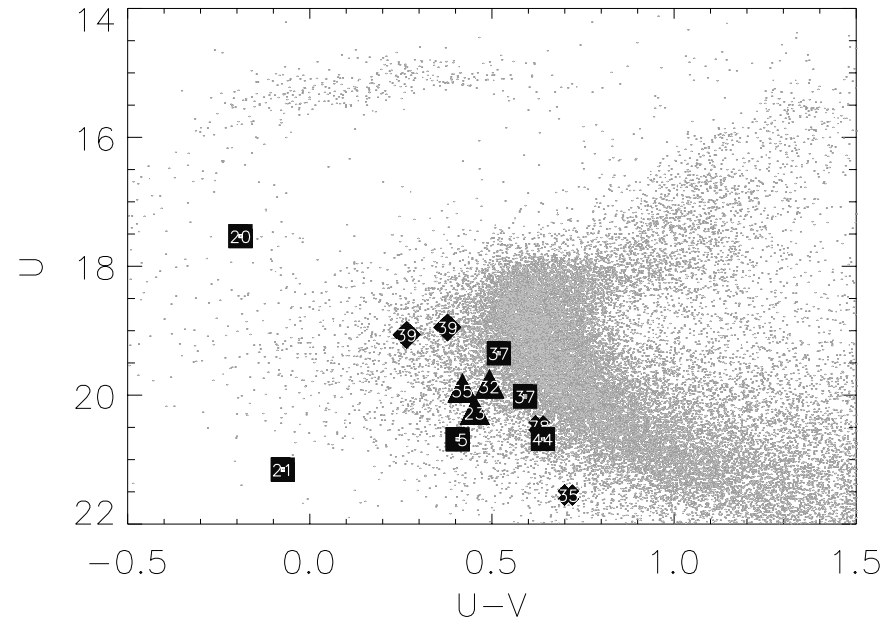

Fig. 2. The $U, U-V$ diagram of the globular cluster M 22. The diamonds indicate blue optical sources found inside the error circle of an $\mathrm{X}$-ray source in the core, the triangles indicate a blue optical source found inside the error circle of an X-ray source in the half mass radius, the squares indicate a blue optical source found inside the error circle of an X-ray source just outside the half mass-radius and the crosses indicate the blue optical sources found inside the error circle of an $\mathrm{X}$-ray source way outside the half mass-radius (NB: source 78 can just be seen behind source 44). The X-ray source identification number (as given in Table 1) to which the blue source may be an optical counterpart is indicated on the symbol.

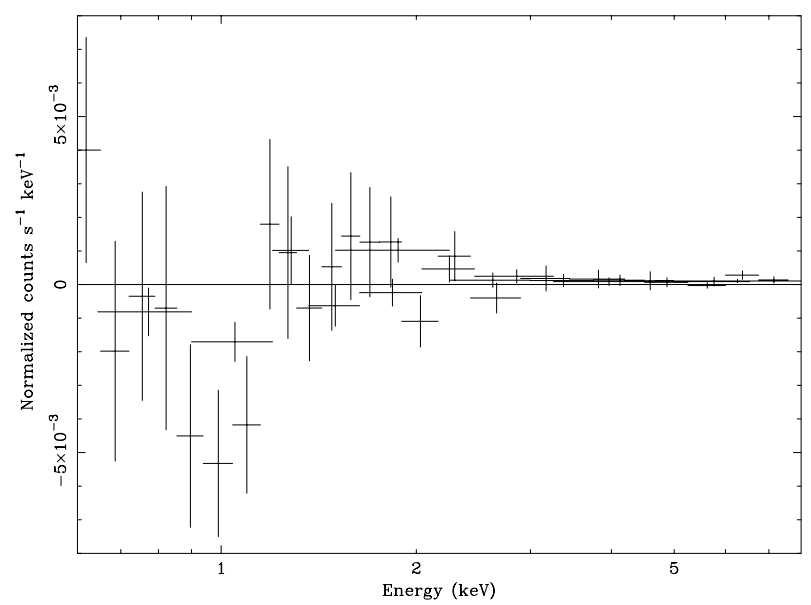

Fig. 3. Residuals after fitting the EPIC spectrum of source 36 with a simple power law model. The region around $1 \mathrm{keV}$ is clearly poorly fit by such a model.

power law, see Table 2, although other simple models such as a thermal bremsstrahlung give a good fit to the data. However, the data consistently over fit the region around $1.0 \mathrm{keV}$, as can be seen in Fig. 3 where we have plotted the residuals resulting from the data minus the power law fit. Using a $\chi^{2}$ test, we find that the region about $1.0 \mathrm{keV}$ deviates from the power law fit at approximately the $2 \sigma$ level. Thus we have tried to fit the spectrum with a simple model (power law) and a Gaussian absorption line. This gives a good fit to the data, see Table 2 and Fig. 4. We are confident that this is not an instrumental effect, as there are no known instrumental edges in the the region of this feature. The absorption feature also appears to be present in

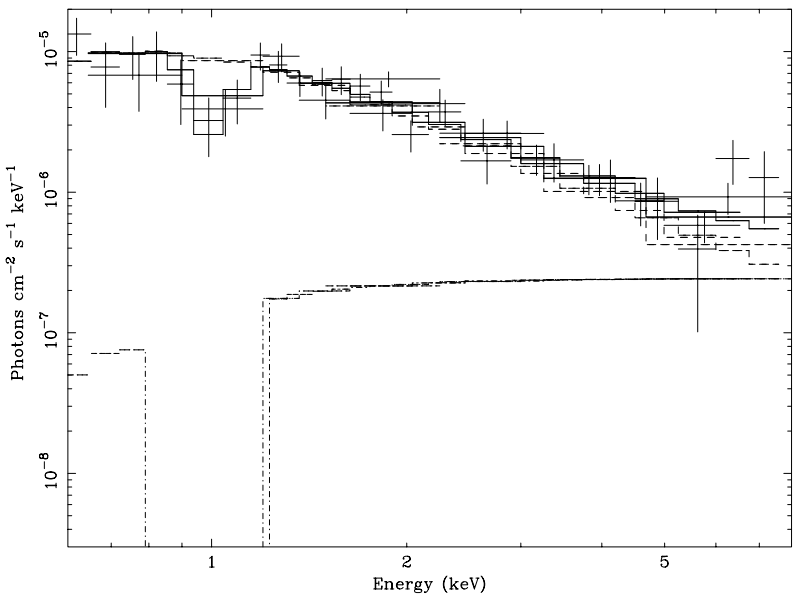

Fig. 4. The EPIC spectrum for source 36, fitted with a power law fit and a Gaussian line (solid line). The two components of this fit are shown as dashed lines.

the data from all three cameras. Such Gaussian lines have been seen in the spectra of some neutron stars e.g. the neutron star pulsar 1E1207.4-5209 (Sanwal et al. 2002; Mereghetti 2002; Bignami et al. 2003) or RBS1223 (1RXS J130848.6+212708) (Haberl et al. 2003), and are thought to be due to resonant cyclotron absorption. Bignami et al. (2003) found three absorption lines at $0.7,1.4$ and $2.1 \mathrm{keV}$, embedded in the continuum, which they fitted with two blackbodies $(k T=0.21$ and $0.40 \mathrm{keV}$ ). Our data can also be well fitted with an absorption line as in Table 2 and two blackbodies $\left(k T_{1}=0.23 \pm\right.$ $0.10 \mathrm{keV}$ and $k T_{2}=0.74 \pm 0.13 \mathrm{keV}, \chi_{v}^{2}=1.17,34$ degrees of freedom). This could indicate that source 36 is similar in nature to 1E1207.4-5209 or RBS1223. If the absorption feature is real and due to cyclotron resonance, we would expect a magnetic field strength of approximately $1.1 \times 10^{11} \mathrm{G}$, if the feature is due to electron cyclotron resonance, as the absorption lines observed in 1E1207.4-5209. Alternatively we would expect a magnetic field strength of approximately $2.1 \times 10^{14} \mathrm{G}$, if the feature is due to proton cyclotron resonance, as the absorption line observed in RBS1223. We have also searched for a pulse period between 0.001-10 s, using Fourier analysis and period folding. However, we find no significant period in this range. This is not surprising, as such an object could be part of a binary system, given the high stellar densities in globular clusters, especially in the cores of GCs. To detect periodicities of a millisecond pulsar in a binary, it is imperative that the orbital motion is taken into account. Thus a pulsation could exist, but we are unable to detect it, as we could be lacking information about a binary orbit. A longer observation may help to confirm these results, in particular to identify whether there is a pulsation. If this source is indeed a neutron star, it is likely that it is a cluster member.

However, a Raymond Smith or a bremsstrahlung model can not be ruled out as the most appropriate model to fit this data. It is unlikely that the Raymond Smith model is the best fit to the data if this source is located in the globular cluster, see Sect. 3, as this model is indicative of emission from hot, diffuse gas, such as that from a galaxy. However, a bremsstrahlung model, with a temperature of $\sim 9 \mathrm{keV}$, gives a good 
description of the data, discarding the possible absorption feature. Such a model is indicative of a cataclysmic variable (dwarf nova) see e.g. Richman (1998), Gendre et al. (2003a), where we expect to find several such sources in the cores of globular clusters. Anderson et al. (2003) found an optically variable source at $18^{\mathrm{h}} 36^{\mathrm{m}} 24^{\mathrm{s}} 66$ and $-23^{\circ} 54^{\prime} 35^{\prime \prime} 5$, which they name CV1. We do not detect this source in our optical data, probably due to the stellar confusion in the centre of the core of the cluster. They postulate that this source is a dwarf nova based on the star's proper motion, $\mathrm{H}_{\alpha}$ emission, variability and brightness in quiescence $\left(V_{606}=18.77\right)$ and they assert that the observed 1999 optical brightening ( $~ 3$ mag, Sahu et al. 2001) over 20-26 days was a dwarf nova outburst. They also note that the source falls within the X-ray error circle of the Rosat source 4 and thus assert that this is the optical counterpart. CV1 is located at the edge of the positional error circle of our X-ray source 36 . However, the light-curve of the alleged dwarf-nova outburst is rather untypical. First, its long rise-time $\sim 10$ days is observed only exceptionally, for example in so-called "anomalous" outbursts of the dwarf nova SS Cyg (Warner 1995, and references therein). Second, its duration is much longer than that of normal outbursts and would suggest a so-called superoutburst, but with a rather low amplitude (observed nevertheless in the superoutburst prototype system SU UMa). In fact, the 1999 outburst light-curve is reminiscent of the optical lightcurves of some X-ray transient event, especially from low-mass binary systems containing neutron stars (see e.g. Chen et al. 1997). If the 1999 event were indeed related to an X-ray outburst it would have been detected by RXTE ASM if its flux was $\gtrsim 2.3 \times 10^{-10} \mathrm{erg} \mathrm{cm}^{-2} \mathrm{~s}^{-1}$ or its luminosity was $\gtrsim 2.5 \times 10^{35} \mathrm{erg} \mathrm{s}^{-1}(2-10 \mathrm{keV})$ at the distance of the cluster. We have obtained the RXTE ASM lightcurve of this source for the whole of 1999. We find no evidence for a burst at the time of the proposed outburst of CV1. However, many cataclysmic variable (CV) X-ray outbursts are fainter than this limit e.g. SS Cyg (Jones \& Watson 1992) as are some X-ray binary outbursts (see e.g. Chen et al. 1997). Therefore these observations can neither confirm nor negate the outburst hypothesis. The association of the 1999 outburst with a neutron-star binary, where in this case the neutron star would be a pulsar, could also imply that the best spectral fit is the power law as in the case of other transient X-ray binaries containing pulsars e.g. IGR J16358-4726 (Patel et al. 2004) or SAX J1808.4-3658 (Campana et al. 2002), with a possible Gaussian absorption feature that could be attributed to cyclotron resonant absorption. Further multiwavelength observations should confirm the true nature of this source.

\subsubsection{Source 39}

This core source is well fitted by a hard power law or by a high temperature thermal bremsstrahlung, see Table 2 and is found to be variable on timescales of hours, see Fig. 5 and Table 2. We find two blue optical sources in the error circle of the X-ray source with $U$-band magnitudes of 18.9 and 19.1 and $U-V$ of 0.4 and 0.3 respectively, see Table 3 . If either of these is the optical counterpart, we find an $L_{\mathrm{x}} / L_{\mathrm{opt}}$ value of $\sim 0.6$, which is

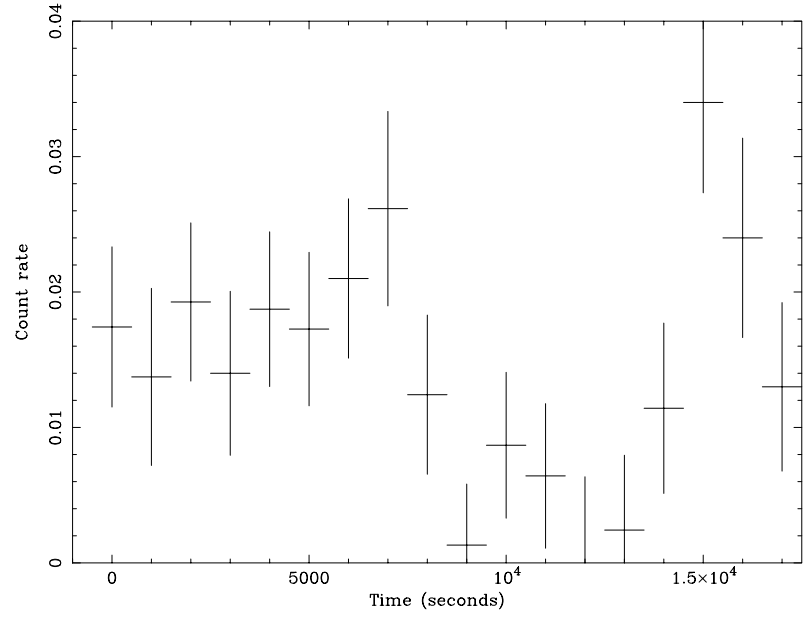

Fig. 5. The PN data lightcurve (binsize = 1000 s) for source 39 .

Table 3. Possible optical counterparts to the M22 X-ray sources.

\begin{tabular}{|c|c|c|c|c|c|}
\hline X-ray & RA & Dec & & $U$ & $(U-V)$ \\
\hline Src. ID & $\mathrm{m}$ & $\circ$ & " & & \\
\hline 20 & 183615.6 & -2351 & 02.4 & $17.53 \pm 0.02$ & $-0.19 \pm 0.03$ \\
\hline 32 & 183619.0 & -2352 & 18.0 & $19.86 \pm 0.03$ & $0.49 \pm 0.05$ \\
\hline 55 & 183614.6 & -23553 & 35.2 & $19.92 \pm 0.04$ & $0.42 \pm 0.07$ \\
\hline 35 & 183530.2 & -23543 & 33.0 & $21.55 \pm 0.06$ & $0.70 \pm 0.07$ \\
\hline 23 & 183628.9 & -23562 & 25.9 & $20.27 \pm 0.05$ & $0.45 \pm 0.07$ \\
\hline 39 & 183625.4 & -2355 & 20.8 & $18.94 \pm 0.05$ & $0.38 \pm 0.08$ \\
\hline 39 & 183625.5 & -2355 & 13.7 & $19.06 \pm 0.03$ & $0.27 \pm 0.08$ \\
\hline 37 & 183638.7 & -2354 & 42.5 & $19.34 \pm 0.03$ & $0.52 \pm 0.05$ \\
\hline 37 & 183638.3 & -2354 & 49.3 & $20.01 \pm 0.03$ & $0.59 \pm 0.05$ \\
\hline 21 & 183645.4 & -23515 & 58.4 & $21.15 \pm 0.05$ & $-0.07 \pm 0.08$ \\
\hline 5 & 183608.1 & -2359 & 20.3 & $20.68 \pm 0.05$ & $0.41 \pm 0.06$ \\
\hline 78 & 183544.5 & -2400 & 10.7 & $20.48 \pm 0.04$ & $0.63 \pm 0.07$ \\
\hline 44 & 183618.5 & -24005 & 54.9 & $20.68 \pm 0.04$ & $0.64 \pm 0.06$ \\
\hline
\end{tabular}

similar to that found for cataclysmic variables (e.g. Schwope et al. 2002; Verbunt et al. 1997). Both the spectrum and the temporal analysis is consistent with this hypothesis, where cataclysmic variables have high temperature bremsstrahlung spectra (e.g. Pooley et al. 2002; Gendre et al. 2003a). Follow up spectroscopy should confirm this hypothesis and identify the optical counterpart.

\subsubsection{Source 33}

The faintest core source is best fitted by a two temperature $\left(1 \times 10^{6} \mathrm{~K}\right.$ and $\left.4 \times 10^{7} \mathrm{~K}\right)$ Raymond Smith model, see Table 2. Dempsey et al. (1993) found, following the analysis of 44 active (RS CVn) binaries observed with Rosat, that a two temperature thermal plasma model, with typical temperatures of $2 \times 10^{6} \mathrm{~K}$ (with a scatter of approximately $1-3 \times 10^{6} \mathrm{~K}$ ) and $1.6 \times 10^{7} \mathrm{~K}$ (with a scatter of approximately $1-4 \times 10^{7} \mathrm{~K}$ ) gave a good description of the RS CVn X-ray spectra. The fact 
that we find no optical counterpart to this source in our photometric dataset could support the hypothesis that this source is an RS CVn binary, where our photometric dataset is designed to detect blue sources and the optical counterparts of RS CVn and BY Dra binaries are red (Dempsey et al. 1997). Source 33's luminosity $\left(4 \times 10^{31} \mathrm{erg} \mathrm{s}^{-1}(0.1-2.4 \mathrm{keV})\right.$ at the distance of the cluster) is also consistent with the RS CVn binary hypothesis (Dempsey et al. 1993).

One would perhaps expect that the X-ray light curve of an active binary would be variable (see e.g. Gendre et al. 2003a), although it is quite probable that during the full $9 \mathrm{~h}$ of this observation no discernible flickering or flaring event occurred, e.g. Osten et al. (2000).

\subsection{Possible cluster members without fitted spectra}

\subsubsection{Sources 5,37 and 55}

Source 37 appears to be well fitted by a low temperature blackbody, see Fig. 6. Such a soft spectrum can indicate a quiescent low mass X-ray binary with a neutron star primary, or a foreground star (see e.g. Gendre et al. 2003a,b). We can rule out the foreground star hypothesis as the radius of such a star with the observed blackbody emission would be several orders of magnitude smaller than the smallest M-dwarf stars. From its position, just outside the half mass-radius, this source could be a member of the globular cluster and thus a quiescent low mass $\mathrm{X}$-ray binary with a neutron star primary. However, its luminosity would then be $1 \times 10^{31} \mathrm{erg} \mathrm{s}^{-1}$, at the lower end of the faintest known quiescent low mass X-ray binary with a neutron star primary, SAX J1808.4-3658 $\left(5 \times 10^{31} \mathrm{erg} \mathrm{s}^{-1}\right.$ Campana et al. 2002). Also, if such systems are formed primarily through collisions, as oppose to from their primordial binaries and thus the relationship between the collision rate and number of neutron star X-ray binaries (Gendre et al. 2003b; Pooley et al. 2003; Heinke et al. 2003) is valid, we do not expect to find any such objects in M 22, due to its low central density and thus low collision rate.

Such a soft spectrum can also be indicative of a millisecond pulsar e.g. Grindlay et al. (2002), where a blackbody of $k T=0.5 \mathrm{keV}$ indicates a temperature of $5.8 \times 10^{6} \mathrm{~K}$, which is consistent with the temperature of the heated polar caps of a millisecond pulsar $\left(10^{6}-10^{7} \mathrm{~K}\right.$ e.g. Zhang \& Cheng 2003; Zavlin \& Pavlov 1998, and references therein). Calculating the radius of the emission area using the blackbody model fit, we find a radius of $0.05 \mathrm{~km}$, which is smaller than the expected radius of emission from polar caps $(\sim 1 \mathrm{~km}$ e.g. Zhang \& Cheng 2003; Zavlin \& Pavlov 1998, and references therein). Zavlin et al. (1996) state however, that blackbody models can produce higher temperatures and smaller sizes due to the fact that the $\mathrm{X}$-ray spectra emerging from light-element atmospheres are harder than blackbody spectra. Indeed Grindlay et al. (2002) found that the radii determined from the blackbody temperatures of the millisecond pulsars in the globular cluster 47 Tuc where also significantly less than the expected radius of $1 \mathrm{~km}$. The X-ray luminosity is also similar to field millisecond pulsars (e.g. Webb et al. 2004). If source 37 is indeed a millisecond pulsar, the optical counterpart suggested in Fig. 2 may not

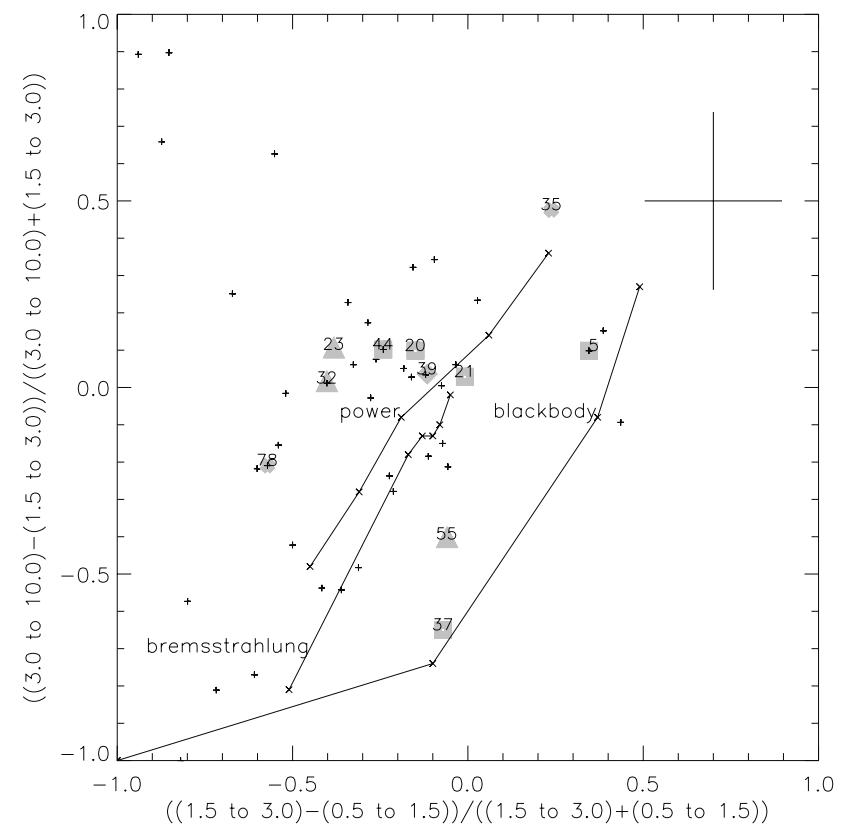

Fig. 6. An X-ray colour diagram of all the detected X-ray sources in $M$ 22. Pluses indicate an X-ray source. Special attention is given to those sources for which a possible optical counterpart has been found. The symbols are the same as those in Fig. 2. The X-ray source identification number is that given in Table 1 . Three lines are indicated showing the colours of a source in the cluster with a power law spectrum, a bremsstrahlung spectrum and a blackbody spectrum. On the power law spectrum line, the crosses (from bottom to top) represent photon indices of 2.5, 2.0, 1.5, 1.0 and 0.5 . On the bremsstrahlung spectrum line, the crosses (from bottom to top) represent temperatures of 1.0, 5.0, 10.0, 15.0, 20.0 and $50.0 \mathrm{keV}$. On the blackbody spectrum line, the crosses (from bottom to top) represent temperatures of 0.1 , $0.5,1.0,1.5 \mathrm{keV}$. For $N_{\mathrm{H}}$ values greater than that of the cluster, the spectral fits move towards the top right. A typical error bar is also shown in the top right hand corner.

be the optical counterpart. Millisecond pulsars are very faint in the optical and could not be detected in the optical observations presented here. Many millisecond pulsars have companions e.g. Lundgren et al. (1996), but they can also be cool red stars, rather than the hot blue stars, which our optical dataset is designed to detect. However, the companion may be close enough that some accretion onto the compact object continues, in the same way as in the accreting millisecond pulsar SAX J1808.4-3658 (Marshall et al. 1998), which could account for the blue nature of the counterpart. Alternatively, the companion star maybe constantly illuminated by the pulsar, as in PSR 1957+20 (Fruchter et al. 1988), which could also account for the blue nature of the counterpart. From the X-ray colours, sources 5 and 55 may also be similar objects.

\subsubsection{Sources 23,44 and 32}

The majority of the proposed optical counterparts $(39,37$, $55,32,44,5$ and 23 ) have $U$-magnitudes between approximately 19 and 20.6 and $(U-V)$ values between 0.35 and 0.6. These correspond to $6.6<M_{U}<8.2$ and $6.2<M_{V}<$ 7.6, values similar to those for cataclysmic variables (see e.g. 
Thorstensen 2003; Szkody et al. 2003). These sources have unabsorbed X-ray fluxes of $8.6 \times 10^{-15}-1.2 \times 10^{-13} \mathrm{erg} \mathrm{cm}^{-2} \mathrm{~s}^{-1}$ $(0.5-10.0 \mathrm{keV})$ or $5.4 \times 10^{-15}-7.3 \times 10^{-14} \mathrm{erg} \mathrm{cm}^{-2} \mathrm{~s}^{-1}$ (0.1-2.4 keV) using PIMMS (Mission Count Rate Simulator) Version 3.4 and a $5 \mathrm{keV}$ bremsstrahlung model. Such values are consistent with those of known cataclysmic variables fluxes. Using a colour-colour diagram to study the X-ray spectral nature of these sources, as the majority do not have enough counts to be able to extract and fit spectra, we find that their X-ray colours, with the exception of sources 5, 37 and 55 which are discussed above, are also similar to known cataclysmic variables. Source 39, which is in the centre of all these sources in Fig. 6, is well fitted by a high temperature bremsstrahlung (see Sect. 5.1.2 and Table 2). Sources 23, 44 and 32 appear to have spectra that are more absorbed than the others. This is nonetheless consistent with a CV spectrum, as CV spectra can be absorbed due to the high intrinsic absorption of the X-rays from the inner disc and/or white dwarfs passing through the edgeon accretion disc e.g. one of the CVs in M 80 (Heinke et al. 2003). All these sources are found in the central regions of the cluster, where we expect to find such binaries, due to mass segregation (e.g. Meylan \& Heggie 1997). Thus if these are the optical counterparts to the X-ray sources, they are likely to be cataclysmic variables and thus members of the cluster.

\subsubsection{Sources 35 and 78}

Sources 35 and 78 have similar colours and luminosities to the aforementioned sources, but they are found towards the edge of the cluster, where we do not expect to find such binaries. However, several close binaries have been found in the outer regions of the globular $\omega$ Centauri see e.g. Gendre et al. (2003a). This has been explained in several ways, including the idea that such binaries may have been ejected through three-body interactions. Thus it is possible that these sources could also be cataclysmic variables and members of the cluster.

\subsubsection{Source 21}

The two other possible optical counterparts in Fig. 2 have $U$, $(U-V)$ magnitudes which are quite different to the other possible optical counterparts. Source 21 has a faint enough $U$-magnitude that it could lie at the top of the white dwarf cooling curve see e.g. Knigge et al. (2002), with a temperature of approximately $80000 \mathrm{~K}$. Its $(U-V)$ value is also consistent with a white dwarf hypothesis (see e.g. Cheselka et al. 1993). However, the X-ray spectrum is too hard for it to be a single white dwarf, see Fig. 6. Single white dwarfs can not have X-ray spectra which peak later than $0.5 \mathrm{keV}$ (see O'Dwyer et al. 2003), thus to have such a hard X-ray spectrum, the white dwarf must have a companion. This companion could either be a late-type active star which is too faint to be observed in the $U, B$ or $V$, but the stellar activity can be detected in X-rays. Alternatively, the hard X-ray emission can be produced through accretion onto the white dwarf from a companion again too faint and/or red to be detected in the optical data, in which case this too is a cataclysmic variable (see e.g. O'Dwyer et al. 2003).

\subsubsection{Source 20}

Source 20 is also interesting due to its position on the colour-magnitude diagram, see Fig. 2. It lies below the blue horizontal branch, home to post-main sequence stars, in the region where we expect blue straggler stars. Blue stragglers are thought to be formed through collisions and thus are more readily formed in globular clusters, where the stellar densities are high. Other blue stragglers have also been detected in X-rays e.g. Belloni \& Tagliaferri (1998); Belloni \& Verbunt (1996), however in all cases, the "hard", faint X-ray emission has been due to a companion star.

\subsubsection{Source 34}

Source 34 is one of the sources that is clustered in the bottom left-hand corner of Fig. 6. These are the softest sources in the cluster. The other two sources are 31 and 17, where source 31 is, from spectral fitting, its extent and location (towards the edge of the field of view) likely to be a background source. Source 17 is also likely to be a background source from its extent and location. However, source 34 is situated very close to the half mass radius and thus could be related to the cluster, in which case, with a luminosity of $3 \times 10^{31} \mathrm{erg} \mathrm{s}^{-1}$ it could be a quiescent low mass X-ray binary with a neutron star primary or a millisecond pulsar, as discussed above for source 37 . Alternatively, this source could be an active binary in the cluster, with temperatures of approximately $1 \times 10^{6}$ and $1 \times 10^{7} \mathrm{~K}$. Its luminosity $\left(3 \times 10^{31} \mathrm{erg} \mathrm{s}^{-1}(0.1-2.4 \mathrm{keV})\right.$ at the distance of the cluster) and lack of optical counterpart could support this theory. From its temperature, this source could also be a latetype active foreground star, but we can rule out this hypothesis as its radius would then be several orders of magnitude smaller than the smallest M-dwarf stars.

\subsection{Non cluster members with fitted spectra}

\subsubsection{Source 30}

This extended source was detected previously by Einstein (Hertz \& Grindlay 1983) and Rosat but with too few counts to be identified. From its distance from the cluster centre and its extent, source 30 is likely to be a background source. Indeed, its X-ray spectrum is best fitted by a MEKAL model, see Table 2 and Fig. 7 or a Raymond Smith model, indicative of emission from a hot diffuse system. Using the distance obtained from the spectral fitting and the extent of the object in our data, we can calculate the radius of the object, which is $0.2-0.4 \mathrm{Mpc}$. This indicates that it could be a giant elliptical galaxy or a cluster of galaxies. However, the temperature is too high for it to be a giant elliptical (O'Sullivan et al. 2001). The luminosity $\left(0.9-3.7 \times 10^{43} \mathrm{erg} \mathrm{s}^{-1}\right)$ and temperature are in good agreement, however, with the luminosity-temperature relationship known for clusters of galaxies, e.g. Babul et al. (2002), thus it is likely that this source is a background object and a galaxy cluster. This is in agreement with Hertz \& Grindlay (1983), who proposed that due to its extent and distance from the cluster core, it could be either a supernova remnant or a cluster of 


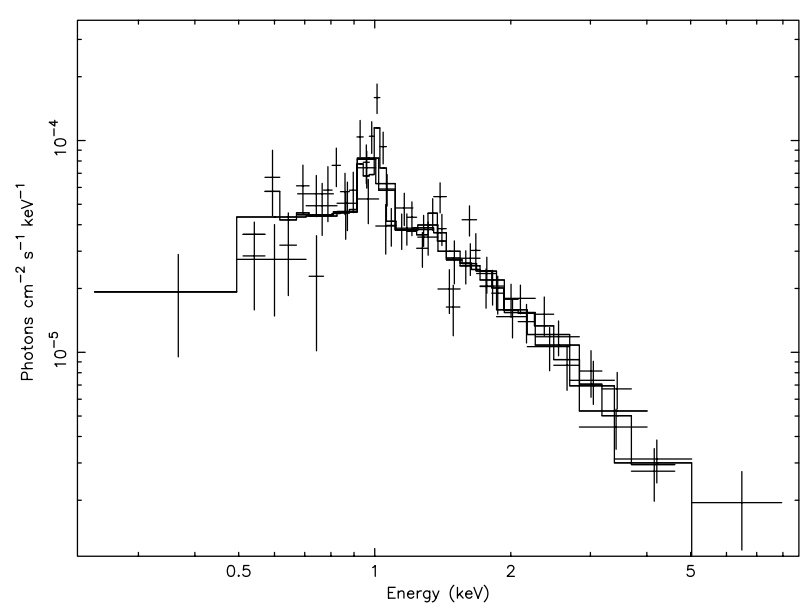

Fig. 7. The EPIC spectrum for source 30, fitted with a MEKAL model.

galaxies. They ruled-out the supernova remnant hypothesis using the fact that the probability of observing such a remnant by chance projection in any field of their survey was $<0.05$, whereas to detect a cluster of galaxies, the probability was more than ten times more likely. They determine a similar luminosity to that determined here, $\left(\sim 0.5-1.0 \times 10^{44} \mathrm{erg} \mathrm{s}^{-1}\right)$, using the assumption that the flux that they observed emanates from the central $500 \mathrm{kpc}$ core of the cluster.

\subsubsection{Other possible background sources without fitted spectra}

From the spectral fitting, the extent of the sources and their location (towards the edge of the field of view), sources 31 and 29 are also likely to be background sources. Sources 31 and 17 are both located in the softest region of the X-ray colour-colour diagram, Fig. 6, and as discussed above source 31 is, from spectral fitting, its extent and location (towards the edge of the field of view) likely to be background source. Source 17 is also likely to be a background source from its extent and location. Sources 42 and 67, which are found in the top left corner of the X-ray colour-colour diagram, see Fig. 6, are also likely to be background sources, as their spectra are very hard, with few counts at lower energies, indicating strong interstellar absorption and hence an origin behind the globular cluster. They are also found towards the edge of the field of view, and thus it is unlikely that they belong to the cluster. Other sources such as 75 and 60, which are located in a similar place to sources 42 and 67 in the X-ray colour-colour diagram and are also positioned towards the edge of the field of view, are also likely to be background sources.

\section{Conclusions}

We have presented both X-ray $(0.2-10.0 \mathrm{keV})$ and optical $(U$, $B$ and $V$ photometry) of the globular cluster M 22. We have shown that $5 \pm 3$ sources belong to the cluster, where these sources are clustered about the centre of the GC. Using the broad band X-ray spectra and X-ray timing analysis, we suggest that two of the three core sources, which are likely to belong to the cluster are either a pulsar and a cataclysmic variable or two cataclysmic variables. We find probable optical counterparts for as many as 11 sources. Using the X-ray and optical data we have presented evidence to show that other sources maybe cataclysmic variables, millisecond pulsars, active binaries or blue stragglers.

The majority of the sources detected in the field of view are background objects. We provide evidence for a cluster of galaxies situated behind M 22 as well as several other galaxies. Planned follow-up spectroscopy of these sources with the VLT UT3 (VIMOS) will help clarify the uncertain nature of these sources.

Acknowledgements. We are indebted to the authors of the WFPRED package at Padova University, in particularly to E. Held. We acknowledge M. Watson for his advice on source 30 . We also thank the anonymous referee for useful comments and suggestions. This article was based on observations obtained with XMM-Newton, an ESA science mission with instruments and contributions directly funded by ESA Member States and NASA. We are also grateful to R. Remillard and all the RXTE ASM team for providing us with the data concerning source 36. The authors N.A.W. and D.B. also acknowledge the CNES for its support in this research. JPL was supported by the CNES and the GDR PCHE.

\section{References}

Anderson, J., Cool, A. M., \& King, I. R. 2003, ApJ, 597, 137

Babul, A., Balogh, M. L., Lewis, G. F., \& Poole, G. B. 2002, MNRAS, 330, 329

Belloni, T., \& Verbunt, F. 1996, A\&A, 305, 806

Belloni, T., \& Tagliaferri, G. 1998, A\&A, 335, 517

Bignami, G. F., Caraveo, P. A., De Luca, A., \& Mereghetti, S. 2003, Nature, 423, 725

Blackburn, J. K. 1995, in Astronomical data analysis software and systems IV, ed. R. A. Shaw, H. E. Payne, \& J. J. E. Hayes (San Francisco: ASP), ASP Conf. Ser., 77, 367

Camilo, F., Lorimer, D. R., Freire, P., Lyne, A. G., \& Manchester, R. N. 2000, ApJ, 535, 975

Campana, S., Stella, L., Gastaldello, F., et al. 2002, ApJ, 575, 15

Carson, J. E., Cool, A. M., \& Grindlay, J. E. 2000, ApJ, 532, 461

Chen, W., Shrader, C. R., \& Livio, M. 1997, ApJ, 491, 312

Cheselka, M., Holberg, J. B., Watkins, R., Collins, J., \& Tweedy, R. W. 1993, AJ, 106, 6

Clement, C. M., Muzzin, A., Dufton, Q., et al. 2001, AJ, 122, 2587

Cool, A. M., Haggard, D., \& Carlin, J. L. 2002, ASP Conf. Ser., 265, 277

Dempsey, R. C., Linsky, J. L., Schmitt, J. H. M. M., \& Fleming, T. A. 1993, ApJ, 413, 333

Dempsey, R. C., Linsky, J. L., Fleming, T. A., \& Schmitt, J. H. M. M. 1997, ApJ, 478, 358

Di Stefano, R., \& Rappaport, S. 1994, ApJ, 423, 274

Djorgovski, S. 1993, ASP Conf. Ser., 50, 373

Edmonds, P. D., Gilliland, R. L., Heinke, C. O., \& Grindlay, J. E. 2003, ApJ, 596, 1177

Fruchter, A. S., Gunn, J. E., Lauer, T. R., \& Dressler, A. 1988, Nature, 334,686

Gendre, B., Barret, D., \& Webb, N. A. 2003a, A\&A, 400, 521

Gendre, B., Barret, D., \& Webb, N. A. 2003b, A\&A, 403, L11 
Grindlay, J. E., Heinke, C., Edmonds, P. D., \& Murray, S. S. 2001, Science, 292, 2290

Grindlay, J., Camilo, F., Heinke, C. O., et al. 2002, ApJ, 581, 470

Haberl, F., Schwope, A. D., Hambaryan, V., Hasinger, G., \& Motch, C. 2003, A\&A, 403, L19

Harris, W. E. 1996, Ap\&SS, 267, 95

Hasinger, G., Altieri, B., Arnaud, M., et al. 2001, A\&A, 365, L45

Heinke, C. O., Grindlay, J. E., Edmonds, P. D., Lloyd, D. A., \& Murray, S. S. 2003, ApJ, 598, 516

Hertz, P., \& Grindlay, J. 1983, ApJ, 275, 105

Hut, P., McMillan, S., Goodman, J., et al. 1992, PASP, 104, 981

Jansen, F., Lumb, D., Altieri, B., et al. 2001, A\&A, 365, L1

Johnston, H. M., Verbunt, F., \& Hasinger, G. 1994, A\&A, 289, 736

Johnston, H. M., \& Verbunt, F. 1996, A\&A, 312, 80

Jones, M. H., \& Watson, M. G. 1992, MNRAS, 257, 633

Kaluzny, J., Kubiak, M., Szymanski, M., et al. 1996, A\&AS, 120, 139

Kaluzny, J., \& Thompson, I. B. 2001, A\&A, 373, 899

Kirsch, M., \& the EPIC Consortium 2002, XMM-SOC-CAL-TN0018

Knigge, C., Zurek, D. R., Shara, M. M., \& Long, K. S. 2002, ApJ, 579, 792

Landolt, A. U. 1992, AJ, 104, 340

Lundgren, S. C., Cordes, J. M., Foster, R. S., Wolszczan, A., \& Camilo, F. 1996, ApJ, 458, 33

Marshall, F. E., Wijnands, R., \& van der Klis, M. 1998, IAUC, 6876

Mereghetti, S., De Luca, A., Caraveo, P. A., et al. 2002, ApJ, 581, 1280

Meylan, G., \& Heggie, D. C. 1997, A\&ARv, 8, 1

Monaco, L., Pancino, E., Ferraro, F. R., \& Bellazzini, M. 2004, MNRAS, 349, 1278

Dwyer, I. J., Chu, Y., Gruendl, R. A., Guerrero, M. A., \& Webbink, R. F. 2003, AJ, 125, 2239

Osten, R. A., Brown, A., Ayres, T. R., et al. 2000, ApJ, 544, 953

O'Sullivan, E., Forbes, D. A., \& Ponman, T. J. 2001, MNRAS, 328, 461

Patel, S. K., Kouveliotou, C., Tennant, A., et al. 2004, ApJ, 602, 45

Peterson, R. C., \& Cudworth, K. M. 1994, ApJ, 420, 612
Pietrukowicz, P., \& Kaluzny, J. 2003, Acta Astron., 53, 371

Pooley, D., Lewin, W. H. G., Homer, L., et al. 2002, ApJ, 569, 405

Pooley, D., Lewin, W. H. G., Anderson, S. F., et al. 2003, ApJ, 591, 131

Portegies-Zwart, S. F., Hut, P., McMillan, S. L. W., \& Verbunt, F. 1997, A\&A, 328, 143

Rasio, F. A., Pfahl, E. D., \& Rappaport, S. 2000, ApJ, 532, 47

Richman, H. R. 1996, ApJ, 462, 404

Rutledge, R. E., Bildsten, L., Brown, E. F., Pavlov, G. G., \& Zavlin, V. E. 2002, ApJ, 578, 405

Sahu, K. C., Casertano, S., Livio, M., et al. 2001, Nature, 411, 1022

Sanwal, D., Pavlov, G. G., Zavlin, V. E., \& Teter, M. A. 2002, ApJ, 574, L61

Schwope, A. D., Brunner, H., Buckley, D., et al. 2002, A\&A, 396, 895

Stetson, P. B. 1987, PASP, 99, 191

Szkody, P., Fraser, O., Silvestri, N., et al. 2003, AJ, 126, 1499

Thorstensen, J. R. 2003, AJ, 126, 3017

Tody, D. 1986, Instrumentation in Astronomy VI, ed. D. L. Crawford, Proc. SPIE, 627, 733

Tody, D. 1993, in Astronomical Data Analysis Software and Systems II, ed. R. J. Hanisch, R. J. V. Brissenden, \& J. Barnes, ASP Conf. Ser., 52, 173

Turner, M. J. L., Abbey, A., Arnaud, M., et al. 2001, A\&A, 365, L27

Valdes, F. G. 1998, in Astronomical data analysis software and systems VII, ed. R. Albrecht, R. N. Hook, \& H. A. Bushouse (San Francisco: ASP), ASP Conf. Ser., 145, 53

Verbunt, F. 2001, A\&A, 368, 137

Verbunt, F., Bunk, W. H., Ritter, H., \& Pfeffermann, E. 1997, A\&A, 327,602

Warner, B. 1995, Cataclysmic Variable Stars (Cambridge: Cambridge University Press)

Webb, N. A., Gendre, B., \& Barret, D. 2002, A\&A, 381, 481

Webb, N. A., Olive, J. F., Barret, D., et al. 2004, A\&A, 419, 269

Zacharias, N., Urban, S. E., Zacharias, M. I., et al. 2000, AJ, 120, 2131

Zavlin, V. E., Pavlov, G. G., \& Shibanov, Y. A. 1996, A\&A, 315, 141

Zavlin, V. E., \& Pavlov, G. G. 1998, A\&A, 329, 583

Zhang, L., \& Cheng, K. S. 2003, A\&A, 398, 639 